\title{
评述 中国糖尿病研究进展专辑
}

\section{我国1型糖尿病的流行病学研究与疾病负担}

\author{
翁建平 ${ }^{*}$
}

中山大学糖尿病研究所, 中山大学广东省糖尿病防治研究中心, 中山大学广东省糖尿病防治重点实验室, 中山大学附属第三医院内分泌与代 谢病学科, 广州 510630

* 联系人, E-mail: wjianp@mail.sysu.edu.cn

收稿日期：2018-01-17; 接受日期：2018-02-28; 网络版发表日期：2018-05-30

摘要 1 型糖尿病是严重影响青少年儿童健康的主要疾病, 也累及相当多的成年人. 我国 1 型糖尿病疾病流行、治 疗现状和卫生经济负担等数据较少, 以至于在制定相关政策时缺乏必需的数据与证据. 研究表明, 我国 1 型糖尿病 患者存在血糖监测频率低、胰岛素治疗不规范、血糖控制差、并发症发生率高、经济负担重等问题。新近完成 的中国1型糖尿病登记注册研究显示，我国全年龄段1型糖尿病发病率为1.01 (95\%置信区间 0.18 1.84)/ 10 万人年, 其中 $0 \sim 14$ 岁, 15 29岁和 $\geq 30$ 岁的发病率分别为 1.93 (0.83 3.03), $1.28(0.45 \sim 2.11)$ 和 $0.69(0.00 \sim 1.51)$. 在新起病患者 中 $65.3 \%$ 为成人. 我国 1 型糖尿病发病率较前明显增加, 治疗现状亟待改进, 疾病负担重. 呼吁社会各界积极支持帮 助 1 型糖尿病患者, 特别是青少年儿童 1 型糖尿病和其家庭, 关心他们的疾病和相关的心理、生理和健康成长 环境.

关键词 1 型糖尿病, 发病率, 疾病负担

1型糖尿病(type 1 diabetes, T1D)是一种自身免疫 性疾病，遗传易感体在病毒感染、药物接触、自身免 疫等多种因素作用下机体免疫功能出现障碍，自身抗 原被激活，最终破坏胰岛 $\beta$ 细胞，血清中胰岛素绝对缺 乏, 从而导致糖尿病发生 ${ }^{[1]}$. 与 2 型糖尿病相比, 典型 1 型糖尿病的特点包括发病年龄较轻, 起病迅速, “三多 一少”等症状明显，常有自发酮症倾向，胰岛素分泌显 著下降甚至缺失，需终身胰岛素治疗维持生命. 由1型 糖尿病导致的代谢紊乱(主要是糖代谢、脂代谢和蛋 白质代谢)对患者特别是青少年儿童生长发育、育龄 妇女妊娠有严重不良影响, 有时甚至危及生命, 因此, 1 型糖尿病是严重威胁青壮年和少年儿童健康的重要 疾病 ${ }^{[2]}$.

\section{1 我国1型糖尿病的发病现状}

根据 20 世纪 90 年代世界卫生组织开展的多国儿童 糖尿病调查项目(multinational project for childhood diabetes, DIAMOND)的调查结果, 我国14岁以下儿童 1 型糖尿病发病率仅为 $0.51 / 10$ 万人年 ${ }^{[3]}$, 是全球发病率 最低的国家之一. 根据以往的推测, 以 1 型糖尿病患者 发病后平均存活20 30年计算, 我国1型糖尿病患者总 例数可能在 $13 \sim 20$ 万左右, 这样的推测其准确性需要 开展全国规模的流行病学调查以证实和明确 ${ }^{[4]}$. 与 1 型 糖尿病较为高发的西方国家相比, 由于缺乏全国1型糖 尿病患者的病例登记和管理制度, 我国在 1 型糖尿病领 域的流行病学和临床研究较为滞后: 现有的大规模 1 型

引用格式: 翁建平. 我国1型糖尿病的流行病学研究与疾病负担. 中国科学: 生命科学, 2018, 48: 834-839

Weng J P. The epidemic study and burden of type 1 diabetes in China (in Chinese). Sci Sin Vitae, 2018, 48: 834-839, doi: 10.1360/N052018-00016 
糖尿病流行病学调查仅有 20 年前开展的针对儿童 1 型 糖尿病患者的DIAMOND项目的中国部分; 临床研究 亦相对较少且样本量小, 卫生经济学方面研究缺乏. 因此，目前对该病的基本信息，如好发人群、危险因 素、血糖控制、并发症现况和治疗管理情况等了解较 少或较不完整. 此外, 现有的少数 1 型糖尿病研究多针 对青少年儿童患者，缺乏成年1型糖尿病患者的资料. 上述 1 型糖尿病相关的基本流行病学资料的缺乏，给 疾病管理带来较大困难，同时也往往使卫生行政部门 制订相应的卫生保健政策时缺乏依据. 目前，在有些 管理部门出台的升学和择业健康体检条例中明确列出 患有 1 型糖尿病为不合格. 因此，从有利于管理患者和 保护患者个人权益角度出发, 有必要在我国建立起1型 糖尿病登记报告制度，并在其基础上开展相关的基础 和临床研究 ${ }^{[2]}$. 在此背景下, 开展了中国1型糖尿病登 记注册研究(the Epidemiological Study of Type 1 Diabetes Mellitus in China, T1D China $)^{[5]}$, 该研究首次 在全国层面开展基于人群的注册研究，以调查中国各 地区、各年龄层的 1 型糖尿病发病率。该研究不仅更 新了我国 1 型糖尿病在青少年儿童中的发病率数据, 也填补了 1 型糖尿病在成人中发病的数据空白. 以下对 该研究作一介绍.

自2010 2013年，中国1型糖尿病登记注册研究共 在中国7个行政区域进行登记注册，调查区域涵盖了 不同地理位置、生活环境和经济发展水平的地区。研 究根据我国行政区域划分在7个行政区域中每个区域 至少选取 1 个代表性地区, 共计 13 个地区, 具体包括: 东 北(哈尔滨、沈阳)、华北(北京)、西北(银川、兰州、 西安)、华东(济南、南京、上海)、西南(成都)、中南 (武汉、长沙)、华南(广州). 通过中国人口普查数据, 确定2010 2013年上述13个地区的常住人口基数. 通 过以下 4 种方式收集 1 型糖尿病患者数据: (i ) 13 个地 区共 505家医院诊疗记录; (ii) 13 个城市228家三甲医 院门诊药房登记系统; (iii) 政府医疗保险数据库; (iv) 患者自我线上及线下登记. 研究者收集详细的患者数 据，由内分泌科和儿科医生组成的专家委员会进行病 例确认后，利用捕获-再捕获(capture-recapture)模型进 行发病率估计.

研究共确认5018例2010 2013年新诊断的1型糖尿 病, 在校正2010 2013年总人口变化后, 13个地区的1型 糖尿病全年龄层发病率预计为 $0.93 / 10$ 万人年 $($ 为方便
起见, 以下发病率数据均省略单位 10 万人年), 其中男 性略高于女性(男性 0.93 , 女性 $0.81, P<0.001$ ). 按年龄 分层发病率分别为：0 14岁组1.90, 15 29岁组 1.02 , $\geq 30$ 岁组 0.51 , 其中 $0 \sim 14$ 岁组男性发病率低于女性(男 性1.72, 女性 $2.21, P<0.001), \geq 15$ 岁组男性发病率则较 高(男性 0.96 , 女性 $0.70, P<0.001$ ). 不同城市全年龄层 1 型糖尿病发病率中，以成都最低(0.52)，兰州最高 (1.57)。0 14岁组发病率以成都最低(1.14), 哈尔滨最 高 (3.59), 且该年龄层发病率与纬度呈正相关 $(P<0.001) .15 \sim 29$ 岁组发病率以银川最低 $(0.14)$, 哈尔 滨最高(2.43). $\geq 30$ 岁组发病率以银川最低(0.03), 武汉 最高(0.77). 15 29岁组和 $\geq 30$ 岁组中并未发现发病率 与纬度存在统计学差异(15 29岁组: $P=0.09 ; \geq 30$ 岁组: $P=0.92)$. 相关分析还发现年龄层、纬度、日照暴露均 与发病率相关. 此外, 研究还分析了来源于诊疗记录的 4661名新诊断 1 型糖尿病患者诊断时的临床特征, 其中 1143 名患者年龄在0 14岁，1664名患者年龄在15 29 岁, 1854 名患者年龄 $\geq 30$ 岁. 结果提示, 年龄越大, 起病 时使用胰岛素治疗的比例越低(0 14岁: 98.4\%; 15 29 岁: $93.5 \%$; $\geq 30$ 岁: $91.5 \% ; P<0.001)$, 但糖尿病酮症和 糖尿病酮症酸中毒的发生率则随着年龄增加而逐渐减 低(糖尿病酮症: 0 14岁: 92.9\%; 15 29岁: 89.0\%; $\geq 30$ 岁： $83.8 \% ; P<0.001$; 糖尿病酮症酸中毒: $0 \sim 14$ 岁: 51.4\%; 15 29岁: 43.0\%; $\geq 30$ 岁: $30.8 \% ; P<0.001)$. C肽 低于检出限 $(<0.2 \mathrm{ng} / \mathrm{mL})$ 的比例在各年龄层中存在统 计学差异(0 14岁：22.5\%；15 29岁：21.7\%; $\geq 30$ 岁: $27.5 \% ; P<0.001$ ), 而糖尿病自身抗体(包括GAD-Ab, IA-2Ab，ICA，IAA，ZnT8A)阳性的比例虽有随年龄增 加而递减的趋势, 但不存在统计学差异 $(0 \sim 14$ 岁: 73.5\%; 15 29岁: 59.5\%; $\geq 30$ 岁: 55.4\%; $P=0.22$ ).

该研究是我国首个全国全年龄层的 1 型糖尿病发 病率研究, 虽然与 20 年前DIAMOND研究 14 岁以下儿 童 $0.51 / 10$ 万人年的发病率相比，该研究 $0 \sim 14$ 岁组 $1.90 /$ 10 万人年的发病率明显升高，但仍处于世界低发病率 水平. 在该年龄层中, 研究者还观察到了纬度与发病 率的正相关性，这一发现是国内外第一个在同一种族 中发现儿童 1 型糖尿病的发病与纬度相关. EURODIAB研究报道了在欧洲多个国家的不同人群中类似 的发现 ${ }^{[6]}$, 美国的SEARCH研究并未得出这一结果 ${ }^{[7]}$. 本研究组推测, 紫外线强度、病毒分布的差异等因素 参与了 1 型糖尿病的发病. 值得重视的是，同为高纬度 
地区的芬兰 $\left(\right.$ 北纬 $60.1^{\circ}$ )和哈尔滨 $\left(\right.$ 北纬 $\left.45.8^{\circ}\right)$, 两地区 间的发病率仍存在明显差异(芬兰 $64.9 / 10$ 万人年，哈尔 滨 $3.59 / 10$ 万人年 $)^{[8]}$, 提示种族差异(遗传因素)可能在 1 型糖尿病发病中起着更为重要的作用. 从发病趋势来 看，该研究 14 岁以下儿童的发病率约是DIAMOND研 究结果的 3.8 倍, 粗略估计年增长率为 $6.5 \%$, 较国外

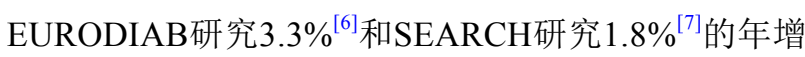
长率相比, 我国14岁以下儿童发病率增长明显较快. 当 然，这可能是由于DIAMOND研究中参与中心报告病 例不全而低估了当时的发病率 ${ }^{[3]}$ ，因此对该增长率的 解读需要谨慎. 尽管中国1型糖尿病登记注册研究证 实中国是世界 1 型糖尿病发病率最低的国家之一，鉴 于中国人口基数庞大， 1 型糖尿病的实际患病人数应 该较多。这次全国1型糖尿病登记注册研究测算我国 每年新发至少 13000 名 1 型糖尿病患者，其中4271名为 儿童. 根据国际糖尿病联盟(International Diabetes Federation, IDF) 2017年世界糖尿病地图的数据, 本研究证 明, 我国新发儿童 1 型糖尿病患者数量在国际上位列第 4 , 排在前3位的依次是美国(14700名)、印度(11300名) 和巴西 $(7600 \text { 名 })^{[9]}$. 此外, 研究中 $65.3 \%$ 的新诊断 1 型糖 尿病患者起病年龄均 $\geq 20$ 岁，其中20 29岁年龄层1型 糖尿病发病率为 $1.15 / 10$ 万人年. 我国的研究在国际上 第一个绘制了 1 型糖尿病流行情况的全景图，为 1 型糖 尿病在成年人的预防与治疗提供了流行病学依据.

\section{2 我国1型糖尿病的疾病负担}

如前述, 在 2010 年以前, 我国关于 1 型糖尿病的流 行病学及临床研究均较少. 2010 年 8 月, 本研究组发起 了广东省1型糖尿病转化医学研究(Guangdong Type 1 Diabetes Mellitus Translational Medicine Study, GTT), 以了解广东省 1 型糖尿病患者现状并在此基础上建立 适合我国国情的 1 型糖尿病管理模式, 从而帮助医务工 作者更好地管理这些患者, 并向政府部门提供我国1型 糖尿病患者的信息资料，以改善这些患者的卫生保健 体系. 目前在广东省内共设立了16家中心，均为各地 级市最具影响力的内分泌专科, 并纳入了全省 21 个地 级市的 100 余家三级和二级医院. 本研究分若干阶段 完成. 已完成的第1阶段是初步建立 1 型糖尿病的登记 报告制度，收集1型糖尿病患者的临床资料，建立包括 血、尿标本在内的标本库. 正在进行的第2阶段是针对
参加第 1 阶段的 1 型糖尿病患者进行定期的随访、观 察，并尝试采用包括课堂教育、报纸、手机和即时通 信平台等在内的多种手段开展糖尿病教育和管理工 作. 同时进行的第3阶段则是在前 2 个阶段的基础上, 逐 步开展相关的临床研究, 探索新技术、新疗法在 1 型糖 尿病中的应用. 本研究是迄今为止国内最大样本量的 1 型糖尿病研究, 即将走入第 8 个年头. 通过对这些患者 的随访观察, 发现了中国1型糖尿病患者具有一些独特 的人口学和临床特征, 以及存在的诸多问题, 如常见成 年后起病、体型偏瘦、血糖监测频率低、胰岛素治疗 方案不规范、血糖控制差以及各种急、慢性并发症频 发等 ${ }^{[2]}$. 这些初步研究结果提示, 有必要采取有效措施 改善我国1型糖尿病患者的治疗和管理方案, 积极规范 地开展新技术、新疗法的研究并转化应用于临床. 下 面对广东省 1 型糖尿病转化医学研究及国内外相关研 究成果作一介绍.

\section{1 常见成年后起病}

GTT研究曾调查了2010 2011年815名1型糖尿病 患者的横断面情况, 结果发现患者年龄的中位数(四分 位间距，下同)为29.6岁(20.3 41.3岁), 发病年龄为 25.3 岁(15.7 35.5岁), 病程为3.3年(1.0 7.3年 $)^{[10]}$. 另一项横 断面研究——国1型糖尿病发病、管理及经济负担 研究(Study of Coverage, Cost, and Care, 3C)调查了 2011 2012年849名北京和汕头两地1型糖尿病患者的 横断面情况, 结果发现患者年龄的中位数为 22 岁 (13 34岁), 其中 $48.4 \%$ 患者病程不超过 6 年 ${ }^{[11]}$.

\section{2 血糖管理不达标}

GTT研究发现，广东省 1 型糖尿病患者的糖化血 红蛋白(hemoglobin $\mathrm{A} 1 \mathrm{c}, \mathrm{HbA} 1 \mathrm{c}$ ) 的中位数为 $8.6 \%$ $(6.9 \% \sim 11.0 \%)$ ，按各年龄段的血糖控制目标计算， $\mathrm{HbA1c}$ 达标患者仅 234 例, 占 $27.5 \%$, 其中13 19岁患者 达标率最低, 仅为 $15.5 \%$, 多因素分析还提示 $13 \sim 19$ 岁 患者、家庭年收入低、未控制饮食、未接受糖尿病教 育、病程短的患者血糖控制差 ${ }^{[10]}$. 虽然与接近 20 年前 的Diabcare-Asia研究中部分中国1型糖尿病患者 10\% 的达标率 $(\mathrm{HbA} 1 \mathrm{c}<7 \%)$ 相比 ${ }^{[12]}$, 血糖控制情况有所改 善, 但较2009年SEARCH研究报道的 $44.4 \%$ 达标率(采 用ADA标准)仍有较大差距 ${ }^{[13]}$. $3 \mathrm{C}$ 研究调查了北京和 汕头两地 1 型糖尿病患者的 $\mathrm{HbA} 1 \mathrm{c}$ 控制情况, 其结果与 
广东省 1 型糖尿病转化医学研究相近, 糖化血红蛋白的 中位数为 $8.5 \%(7.2 \% \sim 10.5 \%)$, 且存在地区差异, 北京 的血糖控制情况优于汕头 $(P=0.002)^{[11]}$.

$3 \mathrm{C}$ 研究还发现，约 $45 \%$ 的患者每日注射 2 次胰岛 素，34.7\%患者每日注射4次胰岛素. 北京患者使用最 多的是中效/短效胰岛素, 而汕头患者使用最多的是预 混胰岛素. 约 $40 \%$ 患者每日监测血糖, 每周监测血糖天 数的中位数为 $3.0(1.0 \sim 7.0)$. 北京患者每日用 $1.5 \sim 3.1$ 条 试纸, 而汕头患者平均每日用 0.9 条试纸. 北京至少 $1 / 5$ 的患者在过去 1 年中没有复查 $\mathrm{HbA} 1 \mathrm{c}$, 而汕头的这一数 字则接近 $2 / 5$, 在这些复查 $\mathrm{HbA} 1 \mathrm{c}$ 的患者中, 绝大多数 患者在过去 1 年仅复查了 1 次 ${ }^{[1]}$ ，与指南推荐每年复查 至少 3 次 $\mathrm{HbA} 1 \mathrm{c}$ 相差较大 ${ }^{[14-16]}$.

\section{3 急慢性并发症发病率高}

(1) 严重低血糖. GTT研究共调查611例1型糖尿病 患者, 共有 175 例患者发生了 403 次严重低血糖(需他人 帮助摄入碳水化合物、静脉输注葡萄糖、应用胰高糖 素等措施方能恢复的低血糖，或血糖值低于 $2.8 \mathrm{mmol} / \mathrm{L}$ ), 严重低血糖发病率为 70.6 次 $/ 100$ 人年, 其 危险因素包括男性、医保报销比例 $<50 \%$ 、病程长、 未控制饮食、体育锻炼 $<150 \mathrm{~min} /$ 周、合并神经病 变、吸烟和低HbA1c, 且严重低血糖存在易复发的特 点，该研究中严重低血糖发病频率最高达 12 次/年 ${ }^{[17]}$. $3 \mathrm{C}$ 研究发现，严重低血糖在青少年中发病率最高，儿 童中发病率最低，其中约 $1 / 5$ 的青少年在过去 1 年内因 低血糖需要住院 ${ }^{[11]}$.

(2) 糖尿病酮症酸中毒. 在 $3 C$ 研究中, $32.3 \%$ 的患 者起病时表现为糖尿病酮症酸中毒(diabetic ketoacidosis, DKA), 且儿童发生DKA的比例最高 $(41 \%)^{[11]}$. 而 GTT研究纳入 611 例1型糖尿病患者，总共有 122 例1型 糖尿病患者发生了 151 次DKA，DKA发病率为 26.4 次/ 100 人年. 其中发生过 1 次DKA的患者有 99 例, 2 次的有 17例, 3次的有6例, 其危险因素包括女性、医保报销比 例、未控制饮食、吸烟和血糖控制差. 在全部151次 DKA事件中，有52次(34.4\%)集中发生在 $3.8 \%$ 的具有 DKA复发史的患者 ${ }^{[18]}$. T1D China研究发现, 我国全年 龄层 1 型糖尿病患者起病半年内合并DKA的比例达到 $40.1 \%, 0 \sim 14$ 岁年龄层中更是高达 $51.4 \%{ }^{[5]}$, 与国外相关 研究 15\% 30\%的比例相比 ${ }^{[19,20]}$, 我国发生DKA的比例 明显偏高. 以DKA起病可能与起病急骤、患者及医务
人员缺乏对疾病认知有关，但诊断糖尿病半年内发生 的DKA则反映存在糖尿病教育不足、使用胰岛素不 足等问题, 因此, 对 1 型糖尿病和DKA的早诊断、早治 疗及疾病教育显得尤为重要.

(3) 糖尿病肾病. GTT研究共纳入 814 例 1 型糖尿 病患者, 其中白蛋白尿和大量白蛋白尿的患者分别为 134 和 44 例，即 $21.9 \%$ 的患者并发糖尿病肾病，危险因 素包括病程、腰慰比、糖化血红蛋白 ${ }^{[2]}$. 3 C研究发现, 不到 $1 / 2$ 的患者在过去两年有篮查肾脏并发症，约 $10.8 \%$ 的患者记录有肾脏并发症 ${ }^{[1]}$.

（4）其他代谢指标及并发症管理情况. 此外, 3C研 究还发现, 约 $30.7 \%$ 患者过去 1 年未监测血压, 在监测 血压的患者中, 有 $10.6 \%$ 合并高血压, 但接近 $1 / 2$ 患者没 有接受降压治疗. $57.3 \%$ 患者合并高脂血症，其中只有 $11 \%$ 接受降脂治疗. $35.2 \%$ 患者在过去两年有过眼底篮 查，其中有 $7.8 \%$ 患者合并视网膜病变，其中成人患者 比例高达 $13.4 \%$. 约 $25 \%$ 患者在过去两年内接受过 足耖 ${ }^{[11]}$.

\section{4 经济负担重}

我国1型糖尿病的卫生经济学研究较少, 有研究调 查了上海儿童1型糖尿病患儿费用, 结果发现, 平均每 患者每月日常治疗费用246.59元，主要支出为胰岛素 费用, 平均为 171.98 元; 发病后平均每年住院次数 0.46 次, 并且随病程延长, 住院次数亦呈现逐渐增多趋势, 儿童1型糖尿病平均每次住院治疗费用 2139.02 元. 经 统计, 1 型糖尿病直接经济负担每人每年 4000 元左右, 间接经济负担 800 元左右, 且存在重治疗、轻监测的 倾向 ${ }^{[22]}$. 但这一研究距今已有 18 年, 有必要调查我国 目前 1 型糖尿病的经济支出, 以了解其疾病负担, 为医 疗政策制定者提供依据.

也可以参考国外多国关于 1 型糖尿病经济负担的 研究结果. 美国一项研究发现, 约 $4 \%$ 的 1 型糖尿病患者 因严重低血糖至少住院一次，平均住院日1.7天，花费 3551 美元, 且其中 $1 / 3$ 患者因为严重低血糖而再次住院. 严重低血糖不仅给患者带来昂贵的住院花费，还会使 出院后每月平均治疗费用增加至少 712 美元 ${ }^{[23]}$. 欧洲 的研究提示, 1 型糖尿病患者健康状况更差, 生活质量 更低, 有更多的误工、失业情况, 需要使用更多的医疗 资源, 且并发症越多状况越严重 ${ }^{[24]}$. 墨西哥一项研究调 查了青少年和儿童 1 型糖尿病家庭用于治疗和监测的 
平均花费，结果为 1689.87 美元/年，其中包括了政府在 门诊及住院时承担的部分. 家庭自费的部分预计 4.06 美元/天，考虑到当地最低工资约4美元/天，因1型糖尿 病自费的部分比例仍高. 其中花费最多的部分是血糖 监测 $(53.0 \%)$, 胰岛素支出占 $14.8 \%{ }^{[25]}$. 巴西的全国多中 心研究发现，巴西 1 型糖尿病患者人均直接医疗成本为 1319.15 美元/年，其中 $92.20 \%$ 为治疗相关费用(人均 1216.33 美元/年), 胰岛素和自我血糖监测支出占 $52.82 \%$ (人均 696.78 美元/年) ${ }^{[26]}$. 苏丹的 1 型糖尿病患儿 家庭收入中位数为每年1222美元，而每年用于 1 型糖尿 病管理的支出为 283 美元，其中胰岛素占支出的 $36 \%$.
在家庭用于健康的总支出中, $65 \%$ 用于 1 型糖尿病 ${ }^{[27]}$.

以上一系列研究结果揭示, 我国1型糖尿病治疗现 状不容乐观. 事实上综合管理有助于 1 型糖尿病血糖控 制，包括免费提供血糖仪及试纸、定期检测HbA1c、 专科医生护士规律随访、制定个体化治疗方案、建立 稳定医患关系、强化糖尿病教育、多学科协作等 ${ }^{[28]}$. 在我国现行的医疗卫生保健体系下, 1 型糖尿病患者的 管理除了需要医务工作者的努力外, 还需要公共卫生 学家在设计大规模流行病学研究方面的帮助和医药卫 生企业的资金投入, 以及更重要的是, 政府部门在医疗 卫生保健政策方面的支持.

\section{参考文献}

1 迟毓婧, 李晶, 管又飞, 等. 1型糖尿病治疗方法的回顾与展望. 生理科学进展, 2010, 41: 55-58

2 翁建平. 中国1型糖尿病研究现况及未来展望. 广东医学, 2012, 33: 2699-2702

3 DIAMOND Project Group. Incidence and trends of childhood Type 1 diabetes worldwide 1990-1999. Diabetic Med, 2006, 23: 857-866

4 翁建平. 关于 1 型糖尿病的诊断及患者数量的思考. 中华糖尿病杂志, 2013, 5: 385-388

5 Weng J, Zhou Z, Guo L, et al. Incidence of type 1 diabetes in China, 2010-13: population based study. BMJ, 2018, 360: j5295

6 Patterson C C, Dahlquist G G, Gyürüs E, et al. Incidence trends for childhood type 1 diabetes in Europe during 1989-2003 and predicted new cases 2005-20: a multicentre prospective registration study. Lancet, 1989, 373: 2027-2033

7 Mayer-Davis E J, Lawrence J M, Dabelea D, et al. Incidence trends of type 1 and type 2 diabetes among youths, 2002-2012. N Engl J Med, 2017, 376: $1419-1429$

8 Harjutsalo V, Sund R, Knip M, et al. Incidence of type 1 diabetes in finland. JAMA, 2013, 310: 427-428

9 International Diabetes Federation. International Diabetes Federation. Diabetes Atlas. 8th ed. 2017

10 邓洪容, 武革, 罗国春, 等. 广东省1型糖尿病患者血糖控制现状及相关因素的横断面调查. 中华医学杂志, 2011, 91: 3257-3261

11 McGuire H C, Ji L, Kissimova-Skarbek K, et al. Type 1 diabetes mellitus care and education in China: The 3C study of coverage, cost, and care in Beijing and Shantou. Diabetes Res Clin Pract, 2017, 129: 32-42

12 Nitiyanant W, Tandhanand S, Mabtab H, et al. The DiabcareAsia 1998 study—outcomes on control and complications in type 1and type 2 diabetic patients. Curr Med Res Opin, 2002, 18: 317-327

13 Petitti D B, Klingensmith G J, Bell R A, et al. Glycemic control in youth with diabetes: the SEARCH for diabetes in youth study. J Pediatr, 2009, 155: 668-672.e3

14 Hanas R, Donaghue K, Klingensmith G. Global IDF/ISPAD Guideline for Diabetes in Childhood andAdolescence. International Diabetes Federation, 2011

15 American Diabetes Association. Standards of medical care in diabetes-2012. Diabetes Care, 2012, 35: S11-S63 
Rydén A, Sörstadius E, Bergenheim K, et al. The humanistic burden of type 1 diabetes mellitus in Europe: examining health outcomes and the role of complications. PLoS ONE, 2016, 11: e0164977

25 Altamirano-Bustamante N, Islas-Ortega L, Robles-Valdes C, et al. Economic family burden of metabolic control in children and adolescents with type 1 diabetes mellitus. J Pediat Endocrinol Metab, 2008, 21: 1163-1168

26 Cobas R A, Ferraz M B, Matheus A S M, et al. The cost of type 1 diabetes: a nationwide multicentre study in Brazil. Bull World Health Organ, 2013, 91: 434-440

27 Elrayah H, Eltom M, Bedri A, et al. Economic burden on families of childhood type 1 diabetes in urban Sudan. Diabetes Res Clin Pract, 2005, 70: $159-165$

28 邢晓欢, 严晋华, 杨黛稚, 等. 综合管理有助于1型糖尿病血糖控制. 中华糖尿病杂志, 2013, 5: 275-278

\title{
The epidemic study and burden of type 1 diabetes in China
}

\author{
WENG JianPing \\ Department of Endocrinology and Metabolism, the Third Affiliated Hospital of Sun Yat-sen University, Guangdong Provincial Key Laboratory of \\ Diabetology, Research Center for Diabetes Care of Sun Yat-sen University, Guangdong Province, Diabetes Institute of Sun Yat-sen University, \\ Guangzhou 510630, China
}

Type 1 diabetes (T1D) threatens the health of children, adolescents and adults. Little was known on the epidemiological and health economic status of T1D in China, which hindered related policy making. The patients with T1D in China faced challenges including low frequency of blood glucose monitoring, improper insulin administration, poor glycaemic control, high rates of diabetic complications and heavy economic burden. The estimated incidence of T1D per 100000 persons years for all ages in China was 1.01 (95\% confidence interval 0.18 to 1.84). Incidence per 100000 persons years by age group was 1.93 (0.83 to 3.03) for 0 -14 years, 1.28 ( 0.45 to 2.11 ) for $15-29$ years, and 0.69 ( 0.00 to 1.51$)$ for $\geq 30$ years. The proportion of new onset cases in participants aged $\geq 20$ years was $65.3 \%$. We found rapid increase of incidence of T1D in China. The current status of T1D in China was unsatisfactory and the patients were under heavy burden. We should conduct more clinical trials and appeal for support from government or other organizations and to help the patients with T1D.

type 1 diabetes, incidence, burden of disease

doi: $10.1360 / \mathrm{N} 052018-00016$ 